\title{
New Factor Structure of the Tampa Scale for Kinesiophobia in Older Japanese Adults After Lumbar Surgery
}

This article was published in the following Dove Press journal: Journal of Pain Research

\author{
Daisuke Higuchi $\mathbb{D I}^{\prime}$ \\ Yuta Watanabe ${ }^{2}$ \\ Yu Kondo $\mathbb{D}^{2}$ \\ Takahiro Miki $\mathbb{D D}^{2}$ \\ 'Department of Physical Therapy, Faculty \\ of Health Care, Takasaki University of \\ Health and Welfare, Takasaki, Japan; \\ ${ }^{2}$ Department of Rehabilitation, Sapporo \\ Maruyama Orthopedic Hospital, Sapporo, \\ Japan
}

Purpose: The Tampa Scale for Kinesiophobia (TSK) has been used worldwide as a measure of kinesiophobia, but its factor structure in older Japanese adults after lumbar surgery is unknown. The purpose of this study was to fill this research gap by identifying the factors that comprise TSK in older Japanese adults after lumbar surgery.

Patients and Methods: Participants were older Japanese adults who had undergone surgery for lumbar spinal stenosis. Clinicodemographic data, TSK, intensity of low back pain and leg pain, dysesthesia (using an 11-point numerical rating scale), and HRQOL (using the EQ-5D$5 \mathrm{~L})$ were collected. After supplementing the missing values by the multiple assignment method, the hypothetical model of TSK was developed by categorical exploratory factor analysis (weighted least squares method, promax rotation). Confirmatory factor analysis (WLSMV method, promax rotation) was used to compare the hypothetical model and the traditional one-factor and two-factor models. Furthermore, we confirmed the relationship between factors extracted from the hypothetical model and HRQOL, pain, and dysesthesia.

Results: Questionnaires were mailed to 302 individuals, and responses were obtained from 211 (72.4 \pm 4.2 years [range: 65-88]; 115 men and 96 women; 804 \pm 343.1 [380-1531] days after surgery; 137 who had undergone decompression and fixation surgery, 74 who had undergone decompression surgery) (response rate: 69.9\%). The hypothesized model consisted of "somatic focus," "activity avoidance," and "efficacy of physical activities," all of which were highly consistent. The fit of the hypothetical model was slightly inferior to that of the traditional twofactor model, but the hypothetical model met the criteria for fit. Somatic focus in the hypothetical model was significantly associated with HRQOL, pain, and dysesthesia.

Conclusion: In older Japanese adults after lumbar surgery, the goodness of fit of the TSK model was maintained by adding efficacy of physical activities as a third factor to the traditional two factors.

Keywords: kinesiophobia, postoperative pain, confirmatory factor analysis, older Japanese adults

\section{Introduction}

Pain is known to be a symptom strongly affecting adults' cognition and emotions and consequently has a notable impact on physical activity. In Japan, where the aging population is the highest in the world, it is especially important to understand the relationship between pain and physical activity in older adults. In a longitudinal study among older Japanese patients with chronic pain, shorter durations of physical activity were found to be associated with a higher risk of functional disability. ${ }^{1}$ It has also been reported that low physical activity is associated with poor subjective health in older
Correspondence: Daisuke Higuchi Department of Physical Therapy, Faculty of Health Care, Takasaki University of Health and Welfare, 50I Nakaorui-machi, Takasaki-shi, Gunma, 370-0033, Japan

Tel +8I-27-352-|29|

Fax +8I-27-352-1985

Email higuchi-d@takasaki-u.ac.jp 
Japanese women with chronic pain. ${ }^{2}$ However, although it has been reported that it is the perception of pain, ${ }^{3}$ rather than pain itself, that determines physical activity, there has been insufficient evidence on the perception of pain in the older Japanese population.

The fear-avoidance model was proposed by Lethem et al in $1983^{4}$ as a framework to explain the relationship among pain, pain perception (fear), and physical activity. The model poses that when the experience of pain causes fear of pain, adults avoid physical activity. In fact, it has been confirmed that older adults with chronic pain are more inactive and have poorer subjective health than those without chronic pain, and that physical activity in older adults with chronic pain was associated with a fear of movement, also called kinesiophobia. ${ }^{3,5}$ It has further been reported that fear of movement is significantly associated with health-related quality of life (HRQOL) in patients diagnosed with musculoskeletal pain. ${ }^{6}$ Thus, fear of movement is one of the important pain perceptions associated with both pain and physical activity.

Questionnaires such as the Fear-Avoidance Beliefs Questionnaire (FABQ) and the Tampa Scale for Kinesiophobia (TSK) have been developed as measures to assess kinesiophobia. ${ }^{7}$ The FABQ is a scale specific to low back pain, whereas the TSK can be applied to pain in general. For this reason, the TSK is more flexible for clarifying future kinesiophobia toward various pain conditions in older Japanese. A questionnaire related to the TSK is the pain catastrophizing scale, ${ }^{8}$ which assesses irrational thoughts about pain, but the TSK is more likely to be able to assess physical activity more directly, as the model suggests that catastrophizing affects disability through the mediation of fear of movement. ${ }^{9}$

The factor structure, which is one aspect of psychometric properties, of the TSK was originally assumed to comprise one factor, but two-factor ${ }^{10}$ and four-factor ${ }^{11,12}$ structures have now been proposed. Multiple structural models have been compared simultaneously in people with chronic neck or back pain ${ }^{13,14}$ and osteoarthritis, ${ }^{15}$ and the two-factor structure has been found to have the best model fit. However, the difference in fit between the one- and twofactor structures is said to be negligible, ${ }^{16}$ and no firm conclusions have been drawn about the factor structure of the TSK. As such, it has been pointed out that the TSK's construct validity has not yet been fully tested. ${ }^{7}$ In addition, it has been argued that there are cultural differences between Asian and Western populations with regard to kinesiophobia, ${ }^{17}$ and a fuller examination of the Japanese population is needed to better understand the factor structure of the TSK. Clarifying the factor structure of TSK in specific populations of Japanese people will help us understand the process by which pain experiences specific to those populations lead to the avoidance of physical activity and further declines in HRQOL, following the so-called fear-avoidance model, which in turn will lead to the practice of effective interventions.

Lumbar spinal canal stenosis (LSS) is one of the most common pain-causing diseases in the older Japanese population, with an estimated prevalence of $5.7 \%$ and 3.65 million patients between the ages of 40 and $79 .{ }^{18}$ If conservative treatment fails, and the pain and dysesthesia are judged to have a significant impact on daily life, surgery is indicated; however, pain and dysesthesia may be present even after surgery. ${ }^{19}$ Thus, it has been hypothesized that the experience of persistent chronic pain and dysesthesia after surgery may induce kinesiophobia, leading to inactivity and low HRQOL. The use of the TSK has already been analyzed with Western people with LSS; its relationship with pain and HRQOL ${ }^{20}$ and the effect of participation in rehabilitation programs on reducing kinesiophobia ${ }^{21}$ have also been reported, but there is no report on the use of TSK in older patients with postoperative LSS, and so the nature of their kinesiophobia is not well understood. Moreover, the TSK has already been used in Japanese patients with diseases other than LSS such as knee osteoarthritis, ${ }^{15}$ anterior cruciate ligament injuries, ${ }^{17}$ and neck and back injuries due to traffic trauma, ${ }^{22}$ but, in contrast to when it has been used with a Western population, the factor structure of the TSK in Japanese has not been reported. Therefore, clarification of psychometric structures as well as detailed information on the TSK scores in older Japanese adults with LSS after surgery is expected to lead to the development of research on kinesiophobia in older Japanese adults with LSS after surgery.

To fill these research gaps in characteristics of the psychometric structure of the TSK in older Japanese adults with LSS after surgery, we aimed to identify the factors comprising the TSK in older Japanese adults who had undergone surgery for LSS at least 1 year prior, considering that the majority of people who had undergone instrumentation surgery had bone union by 1 year after surgery. ${ }^{23}$

\section{Patients and Methods}

\section{Study Design and Ethical Considerations}

This was an observational study conducted between October 2019 and February 2020 in accordance with the Declaration of Helsinki. The ethical review committees of 
Sapporo Maruyama Orthopedic Hospital and Harunaso Hospital reviewed the research, and approval for the study was obtained from each institution in advance (approval numbers: 000025 and 190,105, respectively). A research description, a consent form, and a withdrawalof-consent form were sent to the subjects along with a questionnaire. We asked respondents to write their names on the questionnaire so that we could combine their responses with their medical records in the hospitals. The participants were asked to return the consent form with the completed questionnaire if they agreed to participate. Even for the participants who completed the questionnaire, we provided the option of deleting their research data in case they submitted a consent withdrawal form.

\section{Participants}

People aged 65 years and older with a diagnosis of LSS, lumbar disc herniation, or lumbar degenerative spondylolisthesis based on radiological and clinical examination, and who had undergone surgery at Sapporo Maruyama Orthopedic Hospital and Harunaso Hospital at least one year ago were included in the study. LSS in this study was identified using the definition of Arnordi et $\mathrm{al}^{24}$ of "any type of narrowing of the spinal canal, nerve root canals (or tunnels), or intervertebral foramina"; therefore, lumbar disc herniation and lumbar degenerative spondylolisthesis were considered a type of LSS.

There were 308 older adults who met the inclusion criteria between December 2015 and January 2019. Five had no known addresses and one was dead; as a result, 302 older adults were sent questionnaires.

\section{Assessments \\ Clinicodemographic Data}

Participants' date of birth, gender, date of surgery, and surgical procedure were extracted from the medical records. Current age was calculated from the date of birth. The number of days from the date of surgery to the date of postmark on the return envelope was taken as the number of days since surgery. Surgical procedures were categorized as decompression and fixation, and decompression.

\section{Kinesiophobia}

To evaluate kinesiophobia, the Japanese version of the TSK was used, ${ }^{25}$ which consists of 17 items rated on a 4-point Likert scale: "strongly disagree (1 point)," "disagree ( 2 points)," "agree ( 3 points)," and "strongly agree
(4 points)." For questions $4,8,12$, and 16 , the scores are reversed. Total scores range from 17 to 68 points, with higher scores indicating a stronger degree of kinesiophobia. TSK's internal consistency, retest reliability, and validity have already been confirmed. ${ }^{22,26}$

\section{Pain and Dysesthesia}

An 11-point numerical rating scale (NRS), where a score of 0 was defined as "no pain (dysesthesia)" and a score of 10 was defined as "unbearable pain (dysesthesia)," was used. Participants were asked to recall their persistent low back pain, leg pain, and dysesthesia related to lumbar surgery in the last month and rate the average intensity of their pain or dysesthesia. Alghadir et $\mathrm{al}^{27}$ assessed the intensity of knee pain on several scales, including the NRS, and demonstrated the validity of the NRS.

\section{HRQOL}

The five-level version of the five-dimensional EuroQol scale (EQ-5D-5L) was used to assess the HRQOL of the participants in the previous month. ${ }^{28}$ This scale comprises five subscales, with one question each: mobility, self-care, usual activities, pain/discomfort, and anxiety/depression, with five levels of subjective health state for each subscale. Respondents rate each item on a Likert scale ranging from level 1 indicating no problem to level 5 indicating the inability to walk or perform self-care/activities, extreme pain, or extreme anxiety/depression, depending on the subscale being assessed. The EQ-5D-5L has been previously validated. $^{29,30}$ The index value is calculated based on the answers to the five questions and ranges from a score of 0 , indicating "a state as bad as being dead" to a score of 1 , indicating "full health"; ${ }^{31}$ the conversion values were available for the Japanese population. ${ }^{32}$

\section{Analytic Procedures}

Missing data were complemented through multiple imputation using the chained equation method. All survey items were submitted to the multiple assignment method, and the number of assignments was set to 10. After reviewing the distribution of scores for the 17 questions comprising the TSK, a categorical exploratory factor analysis (C-EFA) (weighted least squares method, promax rotation) based on polychoric correlations was performed on the 17 questions of the TSK for the development of a hypothetical model of the factor structure of the TSK. Parallel analysis was used to determine the number of factors. If items had factor loadings of less than 0.40 , the items were removed, and the factor 
analysis was performed again. The factor analysis was completed when the factor loadings for all items were above 0.40 . Once the final factors were determined, the reliability coefficients (Cronbach's alpha coefficient) for each factor were checked. Cronbach's alpha coefficient was set to be between 0.70 and 0.95 as an acceptable range. ${ }^{33}$

Subsequently, we conducted confirmatory factor analysis (CFA) (WLSMV method, promax rotation) for each of our hypothetical models as well as the traditional onefactor and two-factor models. ${ }^{10}$ Standardized path coefficients and the fit index of the model (Tucker Lewis index [TLI], root mean square error of approximation [RMSEA], standardized root mean residual [SRMR], expected crossvalidation index [ECVI]) were calculated. A TLI of 0.95 or higher, an RMSEA of 0.06 or lower, and an SRMR of 0.08 or lower were judged to be adequate. ${ }^{34}$ The smaller the value of ECVI, the better the fit of the model.

Finally, Spearman's partial rank correlation coefficients between factors extracted from the TSK, the EQ-5D-5L index value, and scores of pain and dysesthesia were calculated. To calculate the correlation coefficients, age, sex (0: male, 1: female), and days after surgery were used as control variables, referring to the following studies: 1) postoperative course was poor when the patient was over 80 years, ${ }^{35,36}$ 2) being female was a predictor of poor subjective health after lumbar spine surgery, ${ }^{36}$ and 3 ) HRQOL changed with postoperative course. ${ }^{37}$

The surgical procedure (decompression and fixation surgery or decompression surgery) was not employed as a control variable because it has been found to make no difference in long-term outcomes.

$\mathrm{R}$ ver. 4.0 (Foundation for Statistical Computing, Vienna, Austria) was used for statistical analysis, and a risk rate of 5\% was considered significant.

\section{Results}

\section{Participant Characteristics and Data Completion}

A set of questionnaires was mailed to 302 older adults (72.8 \pm 4.5 [range: 65-88] years; 164 men, 138 women; 806.0 \pm 341.3 [380-1537] days after surgery; 189 who had undergone decompression and fixation surgery, 113 who had undergone decompression surgery), and responses were obtained from 221 people (73.2\%). After excluding four people with additional spinal surgery, three with surgery for osteoarthritis of the lower extremities, two with severe rheumatoid arthritis, and one with a recent cardiac pacemaker implantation, 211

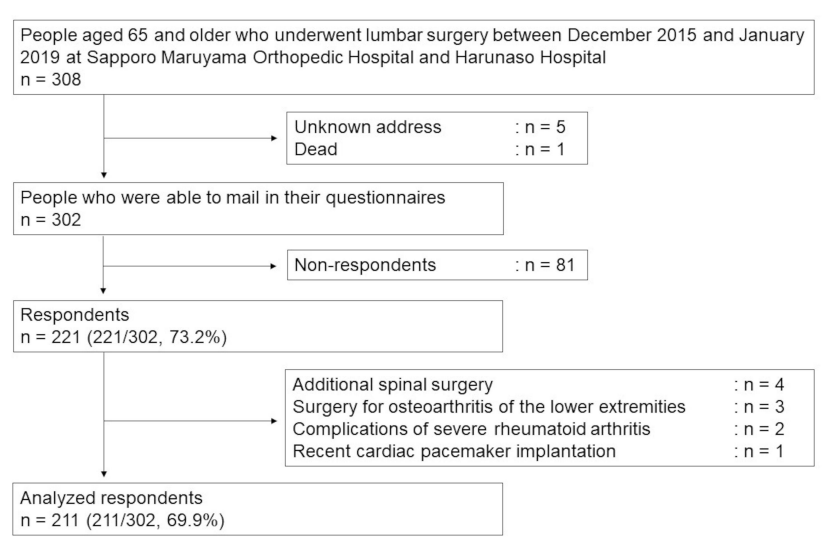

Figure I Flowchart of participants.

people (72.4 4.2 years [range: 65-88]; 115 men and 96 women; $804 \pm 343.1$ [380-1531] days after surgery; 137 who had undergone decompression and fixation surgery, 74 who had undergone decompression surgery) were analyzed (69.9\%) (Figure 1). There were no significant differences in age, sex ratio, postoperative days, or ratio of surgical procedures between respondents and non-respondents.

There were 25 items per respondent in this study (pain and dysesthesia: 3 items, EQ-5D-5L: 5 items, TSK: 17 items); hence, the total number of items answered by all 211 respondents was 5275. As 170 items (3.2\%) were missing, they were supplemented by the multiple assignment method.

\section{Distribution of Scores on the 17 Items of the TSK}

The medians and frequency distributions of the 17 items comprising the TSK are summarized in Table 1. The medians of all items were 2 or 3 points, all items showed an unimodal distribution, and no deviance to minimum or maximum values was observed.

\section{The Development of a Hypothetical Model of the TSK by C-EFA}

Initial parallel analysis suggested that TSK is a three-factor structure (Figure 2). Based on the results of the parallel analysis, the initial C-EFA was conducted and, since the factor loading of item 8 was 0.233 at maximum, item 8 was deleted (left column of Table 2). The three-factor model was still maintained even after item 8 was deleted (Figure 3). In the second C-EFA, the factor loadings for item 1 did not exceed 0.40; therefore, item 1 was also deleted. Again, the three-factor model was maintained (Figure 4). Since the factor loadings for all items exceeded 0.40 in the third 
Table I Medians and Frequency Distributions of the 17 Items in the Tampa Scale for Kinesiophobia

\begin{tabular}{|c|c|c|c|c|c|}
\hline \multirow[t]{2}{*}{ Item } & \multirow[t]{2}{*}{ Median (QD) } & \multicolumn{4}{|c|}{ Proportion (\%) } \\
\hline & & I & 2 & 3 & 4 \\
\hline I & $2(0.5)$ & $40(19.0)$ & $96(45.5)$ & 70 (33.2) & $5(2.4)$ \\
\hline 2 & $2(0)$ & $52(24.6)$ & $128(60.7)$ & $28(13.3)$ & $3(1.4)$ \\
\hline 3 & $2(0)$ & $46(21.8)$ & II (55.5) & $44(20.9)$ & $4(1.9)$ \\
\hline 4 & $2(0.5)$ & $12(5.7)$ & $133(63.0)$ & $50(23.7)$ & $16(7.6)$ \\
\hline 5 & $2(0)$ & $47(22.3)$ & II 5 (54.5) & $46(21.8)$ & $3(1.4)$ \\
\hline 6 & $2(0.5)$ & $39(18.5)$ & $105(49.8)$ & $59(28.0)$ & $8(3.8)$ \\
\hline 7 & $3(0.5)$ & $28(13.3)$ & $66(3 \mid .3)$ & $98(46.4)$ & $19(9.0)$ \\
\hline 8 & $3(0.5)$ & $2(0.9)$ & $70(33.2)$ & III (52.6) & $28(13.3)$ \\
\hline 9 & $3(0.5)$ & $22(10.4)$ & $68(32.2)$ & $113(53.6)$ & $8(3.8)$ \\
\hline 10 & $3(0.5)$ & $19(9.0)$ & $66(31.3)$ & $106(50.2)$ & $20(9.5)$ \\
\hline II & $2(0.5)$ & $42(19.9)$ & II $4(54.0)$ & 49 (23.2) & $6(2.8)$ \\
\hline 12 & $2(0.5)$ & II (5.2) & $132(62.6)$ & $56(26.5)$ & $12(5.7)$ \\
\hline 13 & $3(0.5)$ & II (5.3) & $78(37.0)$ & II 4 (54.0) & $8(3.8)$ \\
\hline 14 & $2(0.5)$ & $21(10.0)$ & 109 (5।.7) & 70 (33.2) & II (5.2) \\
\hline 15 & $2(0.5)$ & $32(15.2)$ & $|2|(57.3)$ & $53(25.1)$ & $5(2.4)$ \\
\hline 16 & $3(0.5)$ & $5(2.4)$ & 88 (4I.7) & $104(49.3)$ & $14(6.6)$ \\
\hline 17 & $3(0)$ & $6(2.8)$ & $45(2 \mid .3)$ & I37 (64.9) & $23(10.9)$ \\
\hline
\end{tabular}

Note: $\mathrm{n}=211$.

Abbreviation: $Q D$, quartile deviation.

C-EFA, this model was adopted as our hypothetical model (right column in Table 2). Following previous studies, ${ }^{10,15,38}$ Factor 1 was designated as "somatic focus" and Factor 2 as "activity avoidance." We named Factor 3 "efficacy of physical activities." The Cronbach's alpha coefficients for factors 1,2 , and 3 increased through the C-EFA iterations, eventually reaching $0.829,0.770$, and 0.705 , respectively. These values met the criteria for internal consistency.

\section{Fitting and Comparison of the Three Models of the TSK by CFA}

The hypothetical model, the traditional one-factor model, and the two-factor model were evaluated by CFA in older Japanese adults after lumbar spine surgery (Table 3). No model had a TLI greater than 0.95. RMSEA was below 0.06 and SRMR was below 0.09 in the hypothetical and two-factor models. The one-factor model did not meet the criteria for any of the fit indices. The ECVI of the two-factor model, the hypothetical model, and the onefactor model were evaluated in decreasing order.

The minimum path coefficient to the observed variable in the one-factor model was 0.047 , while the minimum path coefficients in the hypothetical and two-factor models were -0.402 and 0.390 , respectively, indicating that the observed variables were moderately related to the latent variables. The path coefficients for item 16 belonging to Factor 2 in the hypothetical model and items 16 and 8 in the one-factor model were negative, while the coefficients for the other items were positive.

\section{Relationship Between TSK Factors, and EQ-5D-5L and Pain/Dysesthesia}

The median EQ-5D-5L was 0.740 (quartile deviation: 0.097, range: $0.010-1.000)$. The median NRS scores for low back pain, leg pain, and dysesthesia were 1 (quartile deviation: 1.5 , range: $0-10), 1(1.5,0-8)$, and $1(1.5,0-8)$, respectively. 


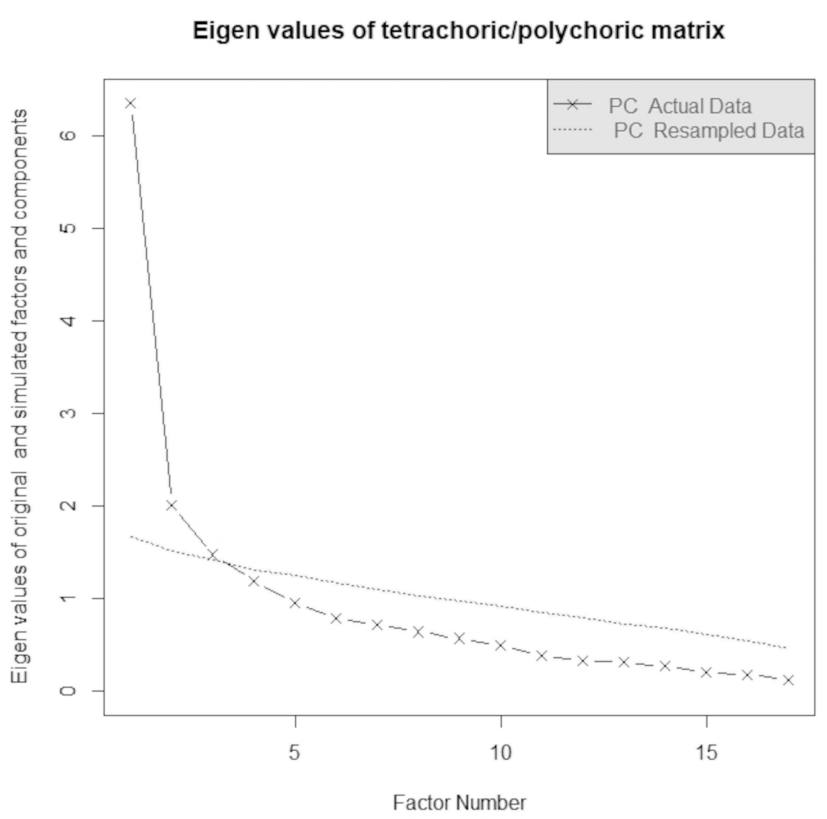

Figure 2 Parallel analysis of the initial categorical exploratory factor analysis.

Since the path coefficient of item 16 belonging to Factor 2 was negative, the total score when item 16 was reversed and the total score when item 16 was deleted were calculated. Spearman's rank correlation coefficients between the total scores of the three TSK factors in the hypothetical model and the index value of EQ-5D-5L as well as the scores of low back pain and leg pain and dysesthesia are summarized in Table 4. The correlation coefficients were adjusted for age, sex, and days after surgery. Factor 1 and Factor 2 showed a significant positive association with the index value of EQ-5D-5L. Factor 3 showed a weak positive effect on EQ-5D-5L, although it was not statistically significant. Factor 1 showed a significant positive association with NRS scores for low back and leg pain and dysesthesia, and Factor 2 showed a significant positive association with NRS scores for low back pain. The correlation coefficients were almost the same when item 16 of Factor 2 was reversed as well as when item 16 was deleted.

\section{Discussion}

\section{Psychometric Structure of the} Hypothetical Model of TSK in Older Japanese Adults After Lumbar Surgery

Items 1 and 8 were deleted during the process of three rounds of C-EFA, resulting in the development of the hypothetical model with a three-factor structure that ensured internal consistency.
Both items 1 and 9 refer to anxiety about physical injury, which belongs to activity avoidance in the traditional two-factor model, while in the hypothetical model, item 1 was deleted and item 9 was assigned to the somatic focus instead of the activity avoidance. This indicates that the activity avoidance in the hypothetical model reduces the presence of anxiety as a reason for activity avoidance compared to that in the two-factor model. Since anxiety is known to have little mediating effect on pain intensity and disability, ${ }^{39}$ it may not be necessary to emphasize anxiety in activity avoidance as it is in the hypothetical model. In addition, item 8 was one of the items that was difficult to translate into Japanese. ${ }^{26}$ Although the appropriateness of the Japanese sentence was assessed by adults and the item eventually passed, it may have been confusing for the older adults to understand. Item 8 did not load strongly with any of the three factors of the hypothesized model; thus, it did not assess any specific construct.

One of the features of the hypothesized model was that the third factor was composed of items 4 and 12, which were deleted in the two-factor model, and we named this factor "efficacy of physical activities." Exercise has been shown to be effective in reducing chronic pain, so-called exercise-induced hypoalgesia (EIH) ${ }^{40,41}$ so it is possible to consider increasing the amount of physical activity in people with chronic pain as a strategy for treating pain. However, EIH is impaired in people with chronic pain; ${ }^{42}$ therefore, encouraging people in pain to continue exercising is a challenge. As the expression of bodyweight exercise behavior has been reported to be influenced by outcome expectations, ${ }^{43}$ it is important for physical therapists in Japan, whose duties include the management and promotion of physical activity and the prescription of therapeutic exercise, to evaluate the expectation that physical activity or exercise will contribute to pain reduction or health improvement.

\section{Validation of the TSK Hypothesis Model and Comparison to Other Traditional Models}

The validity of our hypothetical model as well as the traditional one-factor and two-factor models was tested with CFA. The hypothesized three-factor model and the two-factor model met the criteria for all fit indices except TLI. In contrast, the one-factor model did not meet the criteria for any of the fit indices. The ECVI, where smaller 
Table 2 Factor Loadings, Alpha Coefficients, and Correlation Coefficients for Each Factor of the Tampa Scale for Kinesiophobia

\begin{tabular}{|c|c|c|c|c|c|c|c|c|}
\hline & \multicolumn{4}{|c|}{ Initial Model Constructed with the First C-EFA } & \multicolumn{4}{|c|}{$\begin{array}{l}\text { Modified Model Constructed with the Third } \\
\text { C-EFA }\end{array}$} \\
\hline & Items & Factor I & Factor 2 & Factor 3 & Items & Factor I & Factor 2 & Factor 3 \\
\hline Factor loadings & $\begin{array}{l}3 \\
11 \\
6 \\
5 \\
7 \\
2 \\
9 \\
13 \\
17 \\
14 \\
10 \\
15 \\
16 \\
1 \\
4 \\
12 \\
8\end{array}$ & $\begin{array}{l}\mid .176 \\
0.77 \mid \\
0.701 \\
0.486 \\
0.483 \\
0.463 \\
0.450 \\
-0.179 \\
-0.231 \\
0.135 \\
0.168 \\
0.113 \\
0.056 \\
0.226 \\
0.088 \\
0.074 \\
-0.108\end{array}$ & 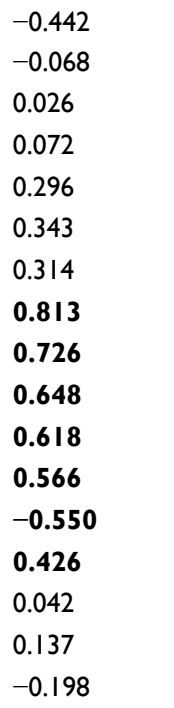 & $\begin{array}{l}0.027 \\
-0.004 \\
-0.044 \\
-0.130 \\
-0.079 \\
0.258 \\
0.101 \\
0.232 \\
-0.054 \\
0.162 \\
0.080 \\
0.020 \\
0.225 \\
0.129 \\
0.785 \\
0.765 \\
0.233\end{array}$ & $\begin{array}{l}3 \\
11 \\
6 \\
7 \\
5 \\
2 \\
9 \\
17 \\
16 \\
13 \\
14 \\
10 \\
15 \\
12 \\
4\end{array}$ & $\begin{array}{l}\mathbf{I} .033 \\
\mathbf{0 . 8 I} \mathbf{I} \\
\mathbf{0 . 7 8 I} \\
\mathbf{0 . 5 5 2} \\
\mathbf{0 . 5 2 7} \\
\mathbf{0 . 4 8 9} \\
\mathbf{0 . 4 8 6} \\
-0.238 \\
0.034 \\
-0.096 \\
0.196 \\
0.245 \\
0.170 \\
\\
0.031 \\
-0.163\end{array}$ & $\begin{array}{l}-0.348 \\
-0.108 \\
-0.027 \\
0.279 \\
0.098 \\
0.202 \\
0.243 \\
\mathbf{0 . 8 3 1} \\
-0.647 \\
\mathbf{0 . 6 4 4} \\
\mathbf{0 . 5 5 0} \\
\mathbf{0 . 5 1 7} \\
\mathbf{0 . 4 9 5} \\
\\
-0.054 \\
-0.085\end{array}$ & $\begin{array}{l}-0.004 \\
0.003 \\
-0.063 \\
-0.011 \\
-0.018 \\
0.282 \\
0.080 \\
-0.011 \\
0.246 \\
0.302 \\
0.196 \\
0.104 \\
0.057 \\
\\
\mathbf{0 . 8 3 6} \\
\mathbf{0 . 7 7 1}\end{array}$ \\
\hline Contribution ratio (cumulative) & & 0.209 & $0.199(0.408)$ & $0.092(0.499)$ & & 0.240 & $0.179(0.420)$ & $0.110(0.529)$ \\
\hline Cronbach's alpha & & 0.829 & 0.780 & 0.489 & & 0.829 & 0.770 & 0.705 \\
\hline Factor correlations & \multicolumn{4}{|c|}{$\begin{array}{l}\text { Factor } 1<>\text { Factor } 2 \\
0.676 \\
\text { Factor } 2<>\text { Factor } 3 \\
0.096 \\
\text { Factor I <> Factor } 3 \\
0.0 .25\end{array}$} & \multicolumn{4}{|c|}{$\begin{array}{l}\text { Factor I <> Factor } 2 \\
0.649 \\
\text { Factor } 2<>\text { Factor } 3 \\
0.347 \\
\text { Factor I <> Factor } 3 \\
0.258\end{array}$} \\
\hline
\end{tabular}

Notes: $\mathrm{n}=211$. Values above 0.40 are in bold.

Abbreviation: C-EFA, categorical explanatory factor analysis.

values indicate a better fit, decreased for the one-factor model, hypothetical model, and two-factor model, in that order. The fact that the one-factor model was not a good fit is in accordance with the transactional model of stress, ${ }^{44}$ which shows that the cognitive process of avoiding physical activity and exercise is not simple.

The hypothetical three-factor model was also statistically acceptable, although the two-factor model is a versatile model that has been shown to be valid not only for the older Japanese adults after lumbar surgery in this study but also for people with chronic low back pain, fibromyalgia, and osteoarthritis. The strengths of the hypothetical model in older Japanese adults after lumbar surgery are as described above, allowing for a positive outcome expectation of physical activity and exercise to be captured along with negative factors such as a sense of inadequacy about their own body and avoidance beliefs about physical activity and exercise. However, there are only two items belonging to the third factor, efficacy of physical activities, and we should point out that uncertainty remains. Therefore, additional validation of the factors of efficacy of physical activities is essential.

The validity of the hypothetical model is discussed by focusing on the differences between the items belonging to somatic focus and activity avoidance in the hypothetical model and the two-factor model. Basically, the items belonging to somatic focus and activity avoidance were similar, but items 2 (low motivation to move due to worsening pain) and 9 (anxiety about accidentally injuring the body) belonged to activity avoidance in the two-factor model, whereas they belonged to somatic focus in the hypothetical model. In our cohort, item 2 was significantly 
Eigen values of tetrachoric/polychoric matrix

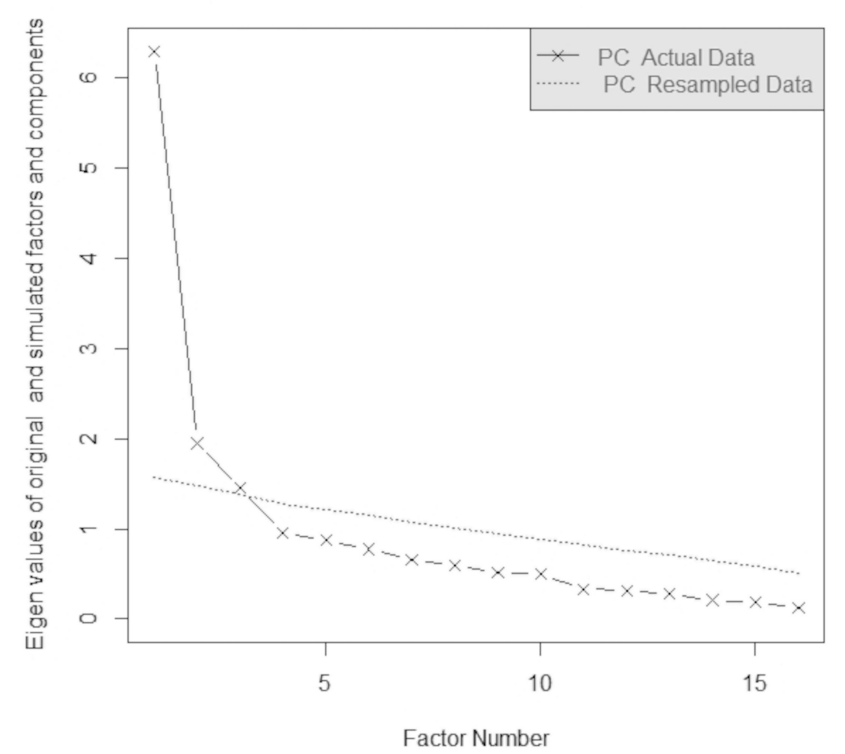

Figure 3 Parallel analysis of the second categorical exploratory factor analysis.

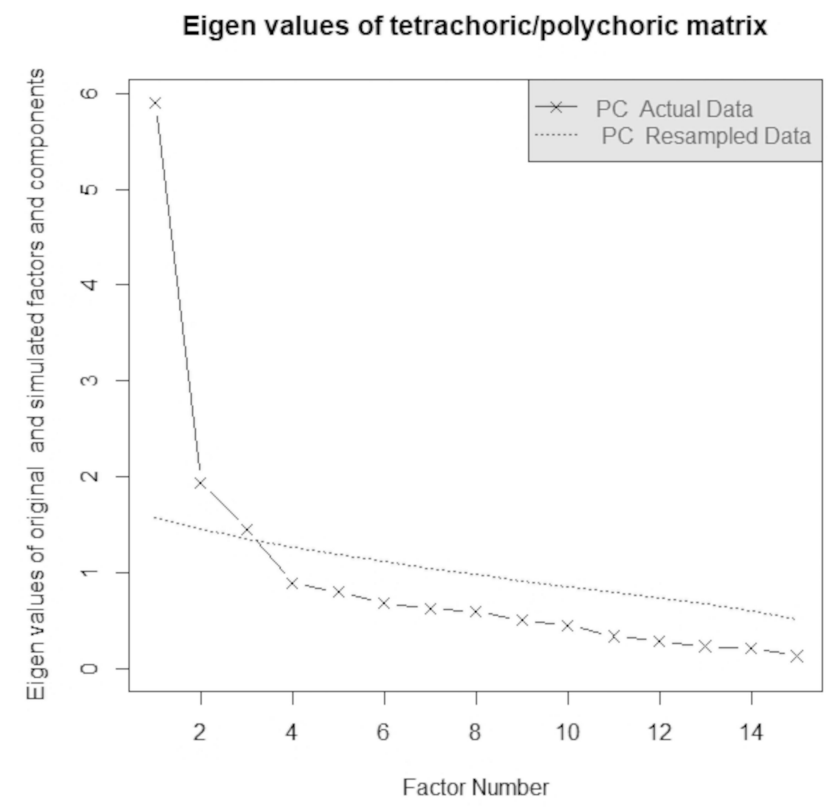

Figure 4 Parallel analysis of the third categorical exploratory factor analysis.

correlated with both other items belonging to somatic focus and activity avoidance (results not shown). Based on this finding, we speculated that item 2 is an ambivalent item, as low motivation to move is likely to be associated with activity avoidance, while the expectation that moving will worsen pain is likely to be associated with somatic focus. Whether the belonging of item 2 to somatic focus is a unique tendency limited to older Japanese adults after lumbar surgery or whether it is due to differences in cultural backgrounds, including language, needs to be further investigated. In addition, the fact that the anxiety asked about in item 9 belonged to somatic focus was in line with research showing that the presence of type D personality, including anxiety tendency, was associated with a risk of low subjective health among older Japanese adults. ${ }^{45}$ Since the report that Japanese university students were more anxious than American students ${ }^{46}$ suggests that there are cultural differences in anxiety tendencies, it is possible that the finding that item 9 belongs to somatic focus is specific to Japanese people.

Another point to be noted in the hypothetical model is that the path coefficient of item 16 (the belief that even if pain is felt, it will not hurt the body), which belongs to activity avoidance, was negative. This means that the strength of activity avoidance belief was paradoxically related to the confidence to cope with pain. It has been found that the reduction of kinesiophobia before and after lumbar surgery is linked to an increase in self-efficacy, ${ }^{47}$ and that there is a positive relationship between selfefficacy and disability in patients with chronic pain and postoperative cardiac patients. ${ }^{48,49} \mathrm{Du}$ et $\mathrm{al}^{50}$ argue that fear-avoidance belief is a risk factor for QOL and selfefficacy is a protective factor. As far as I could find, no research has shown that activity avoidance beliefs coexist with self-efficacy. The fact that the path coefficient of item 16 in activity avoidance was negative may reflect the psychological state specific to older adults who have long since undergone surgery for LSS, but the Japanese text of item 16 may have been difficult for older adults to understand. Even when item 16 was deleted, the correlation between activity avoidance and HRQOL, pain, and abnormal sensation remained almost the same, so it is recommended that item 16 be deleted to make the interpretation of activity avoidance easier.

Among the three factors in the hypothetical model extracted by CFA, somatic focus was the most frequently associated with HRQOL, low back pain, and dysesthesia in our cohort although there are reports that older adults with chronic pain had lower kinesiophobia. ${ }^{51}$ As it has been reported that illness perception was related to HRQOL in patients with fibrous dysplasia, ${ }^{52}$ subjective health is determined by referring to the perception of one's own health status: the stronger the perception of poor health, the stronger the pain and abnormal sensation, and the lower the HRQOL. On the other hand, activity avoidance and efficacy of physical activities were considered to be indirectly related to HRQOL, although they 
Table 3 Estimates and Fit Index of the Three Models According to Confirmatory Factor Analysis

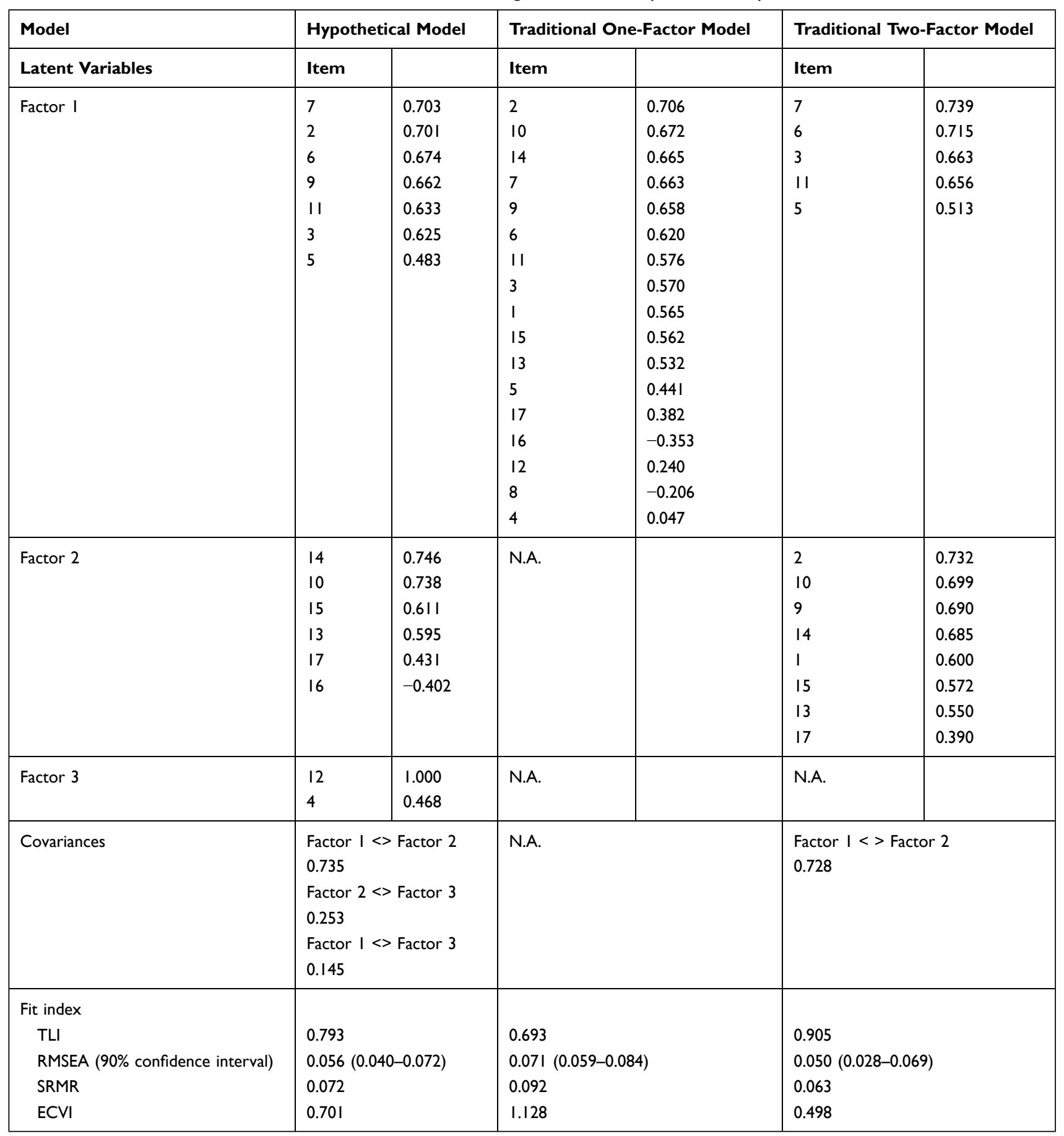

Note: $\mathrm{n}=21 \mathrm{I}$.

Abbreviations: TLI, Tucker Lewis index; RMSEA, root mean square error of approximation; SRMR, standardized root mean residual; ECVI, expected cross-validation index.

were weakly related to HRQOL and pain or dysesthesia. It can be inferred that avoidance beliefs are involved in the background of choosing maladaptive coping as represented by rest (avoidance of physical activity), which is treated by cognitive behavioral therapy and is negatively related to HRQOL. ${ }^{53}$ Furthermore, positive expectation of physical activity is necessary for performing physical activities that are considered to have a positive impact on HRQOL in people with various backgrounds of pain. ${ }^{54-57}$ Therefore, although uncertainty remains regarding the third factor of TSK (efficacy of physical activities), it is important to simultaneously observe the three factors in 
Table 4 Spearman's Partial Rank Correlations Between Factors of the TSK and EQ-5D, Pain, and Dysesthesia

\begin{tabular}{|l|l|l|l|l|}
\hline & Factor I & Factor 2 (R)* & Factor 2 (D)** & Factor 3 \\
\hline EQ-5D & $-0.506(-0.600-0.397)$ & $-0.200(-0.327-0.066)$ & $-0.210(-0.337-0.076)$ & $0.131(-0.005-0.262)$ \\
\hline Low back pain & $0.313(0.185-0.431)$ & $0.146(0.010-0.276)$ & $0.148(0.012-0.278)$ & $-0.114(-0.247-0.022)$ \\
\hline Leg pain & $0.304(0.176-0.423)$ & $0.106(-0.030-0.239)$ & $0.104(-0.033-0.236)$ & $-0.032(-0.167-0.105)$ \\
\hline Leg dysesthesia & $0.351(0.226-0.465)$ & $0.113(-0.023-0.245)$ & $0.117(-0.019-0.249)$ & $-0.015(-0.151-0.121)$ \\
\hline
\end{tabular}

Notes: $\mathrm{n}=2 \mathrm{I} \mathrm{I}$. Age, sex (0: male, I: female), postoperative days, and surgery procedure ( 0 : with fusion; I: with fusion) were used as controlling variables. The values in parentheses represent the $95 \%$ confidence interval. *The total score was calculated by reversing the scores for item 16 . **The total score was calculated by deleting item 16 . Abbreviations: TSK, Tampa Scale for Kinesiophobia; EQ-5D, EuroQol-5 dimensions.

order to broadly understand kinesiophobia in older Japanese adults after lumbar surgery.

\section{Study Limitations and Future Prospects}

This study had two major methodological limitations. First, although the pain and dysesthesia after lumbar surgery reported by the older Japanese adults in this study were likely to be chronic in nature, the duration of the pain and dysesthesia was not collected. Therefore, we cannot conclude that the findings in this study are limited to chronic pain and dysesthesia. Second, as the survey was voluntary, including an optional written questionnaire, there may have been bias in the sample characteristics and responses (i.e., self-selection bias). Moreover, since the respondents were asked to recall their experiences in the most recent month when answering the questions, distortions in the respondents' memory, represented by the phenomenon of telescoping, is another bias that may have affected the results of this study. Thus, the results of this study need to be interpreted cautiously to account for the presence of such biases.

In spite of these limitations, the present study is the first to examine the psychometric structure of TSK in Japanese, especially in older adults after lumbar surgery, and confirmed that the goodness-of-fit of the model did not deteriorate considerably when a new three-factor model, in which efficacy of physical activities was added as a third factor to the traditional two factors of automatic focus and activity avoidance, was adopted for the TSK among older Japanese adults after lumbar surgery. However, there is uncertainty in the third factor, which consists of only two items, and verification is required to compensate for this uncertainty. Traditional somatic focus and activity avoidance were factors that were also valid for older Japanese adults after lumbar surgery; thus, the two factors were accepted internationally regardless of cultural background. Furthermore, among the three factors of the TSK, somatic focus and physical avoidance showed significant negative effects on HRQOL. In this regard, future studies are needed to test the effectiveness of interventions focusing on kinesiophobia to enhance HRQOL among older Japanese adults suffering from pain and dysesthesia after lumbar surgery.

\section{Acknowledgments}

We would like to express our deepest gratitude to Kentaro Tajima (physical therapist, Higashi-Maebashi Orthopaedic Hospital, formerly Harunaso Hospital) for his help with data collection, and Editage for English language editing.

\section{Funding}

This research was funded by JSPS KAKENHI Grant Number JP19K11201. The JSPS was not involved in any part of the study, except for the provision of funds.

\section{Disclosure}

Dr Daisuke Higuchi reports grants from Japan Society for the Promotion of Science (JSPS), during the conduct of the study. The authors declare that they have no other competing interests.

\section{References}

1. Makino K, Lee S, Lee S, et al. Daily physical activity and functional disability incidence in community-dwelling older adults with chronic pain: a prospective cohort study. Pain Med. 2019;20:1702-1710. doi: $10.1093 /$ pm/pny263

2. Kanagae M, Abe Y, Honda S, et al. Determinants of self-rated health among community-dwelling women aged 40 years and over in Japan. Tohoku J Exp Med. 2006;210(1):11-19. doi:10.1620/tjem.210.11

3. Larsson C, Hansson EE, Sundquist K, Jakobsson U. Impact of pain characteristics and fear-avoidance beliefs on physical activity levels among older adults with chronic pain: a population-based, longitudinal study. BMC Geriatr. 2016;16(1):50. doi:10.1186/s12877016-0224-3

4. Lethem J, Slade PD, Troup JD, Bentley B. Outline of a fear-avoidance model of exaggerated pain perception-I. Behav Res Ther. 1983;21:401-408. doi:10.1016/0005-7967(83)90009-8 
5. Kori SH, Miller RP, Todd DD. Kinesiophobia: a new view of chronic pain behavior. Pain Manag. 1990;3:35-43.

6. Goldberg P, Zeppieri G, Bialosky J, et al. Kinesiophobia and its association with health-related quality of life across injury locations. Arch Phys Med Rehabil. 2018;99(1):43-48. doi:10.1016/j. apmr.2017.06.023

7. Lundberg M, Grimby-Ekman A, Verbunt J, Simmonds MJ. Painrelated fear: a critical review of the related measures. Pain Res Treat. 2011;2011:494196. doi:10.1155/2011/494196

8. Elfving B, Andersson T, Grooten WJA. Low levels of physical activity in back pain patients are associated with high levels of fear-avoidance beliefs and pain catastrophizing. Physiother Res Int 2007;12:14-24. doi:10.1002/pri.355

9. Leeuw M, Houben RMA, Severeijns R, Picavet HSJ, Schouten EGW, Vlaeyen JWS. Pain-related fear in low back pain: a prospective study in the general population. Eur J Pain. 2007;11:256-266. doi:10.1016/ j.ejpain.2006.02.009

10. Roelofs J, Goubert L, Peters ML, Vlaeyen JWS, Crombez G. The Tampa Scale for Kinesiophobia: further examination of psychometric properties in patients with chronic low back pain and fibromyalgia. Eur $J$ Pain. 2004;8:495-502. doi:10.1016/j. ejpain.2003.11.016

11. Vlaeyen JW, Kole-Snijders AM, Rotteveel AM, Ruesink R, Heuts PH. The role of fear of movement/(re)injury in pain disability. J Occup Rehabil. 1995;5:235-252. doi:10.1007/ BF02109988

12. Acar S, Savci S, Keskinoğlu P, et al. Tampa Scale of Kinesiophobia for Heart Turkish Version Study: cross-cultural adaptation, exploratory factor analysis, and reliability. J Pain Res. 2016;9:445-451. doi: $10.2147 /$ JPR.S105766

13. Goubert L, Crombez G, Van Damme S, Vlaeyen JWS, Bijttebier P, Roelofs J. Confirmatory factor analysis of the Tampa Scale for Kinesiophobia: invariant two-factor model across low back pain patients and fibromyalgia patients. Clin J Pain. 2004;20:103-110. doi:10.1097/00002508-200403000-00007

14. French DJ, France CR, Vigneau F, French JA, Evans RT. Fear of movement/(re)injury in chronic pain: a psychometric assessment of the original English version of the Tampa Scale for Kinesiophobia (TSK). Pain. 2007;127:42-51. doi:10.1016/j.pain.2006.07.016

15. Heuts PH, Vlaeyen JW, Roelofs J, et al. Pain-related fear and daily functioning in patients with osteoarthritis. Pain. 2004;110:228-235. doi:10.1016/j.pain.2004.03.035

16. Larsson C, Hansson EE, Sundquist K, Jakobsson U. Psychometric properties of the Tampa Scale of Kinesiophobia (TSK-11) among older people with chronic pain. Physiother Theory Pract. 2014;30: 421-428. doi:10.3109/09593985.2013.877546

17. Huang H, Nagao M, Arita H, et al. Reproducibility, responsiveness and validation of the Tampa Scale for Kinesiophobia in patients with ACL injuries. Health Qual Life Outcomes. 2019;17:150. doi:10.1186/ s12955-019-1217-7

18. Yabuki S, Fukumori N, Takegami M, et al. Prevalence of lumbar spinal stenosis, using the diagnostic support tool, and correlated factors in Japan: a population-based study. J Orthop Sci. 2013;18: 893-900. doi:10.1007/s00776-013-0455-5

19. Kasukawa Y, Miyakoshi N, Kobayashi T, et al. Limaprost or pregabalin: preoperative and postoperative medication for pain due to lumbar spinal stenosis. Pain Pract. 2018;18(5):625-630. doi:10.11 11/papr.12653

20. Tomkins-Lane CC, Lafave LM, Parnell JA, et al. The spinal stenosis pedometer and nutrition lifestyle intervention (SSPANLI): development and pilot. Spine J. 2015;15:577-586. doi:10.1016/j. spinee.2014.10.015

21. Monticone M, Ferrante S, Teli M, et al. Management of catastrophising and kinesiophobia improves rehabilitation after fusion for lumbar spondylolisthesis and stenosis. A randomised controlled trial. Eur Spine J. 2014;23:87-95. doi:10.1007/s00586-013-2889-z
22. Kikuchi N, Matsudaira K, Sawada T, Oka H. Psychometric properties of the Japanese version of the Tampa Scale for Kinesiophobia (TSK-J) in patients with whiplash neck injury pain and/or low back pain. J Orthop Sci. 2015;20(6):985-992. doi:10.1007/s00776-0150751-3

23. Han SH, Hyun SJ, Jahng TA, Kim KJ. A comparative radiographic analysis of fusion rate between L4-5 and L5-S1 in a single level posterior lumbar interbody fusion. Korean J Spine. 2015;12:60-67. doi:10.14245/kjs.2015.12.2.60

24. Arnoldi CC, Brodsky AE, Cauchoix J, et al. Lumbar spinal stenosis and nerve root entrapment syndromes. Definition and classification. Clin Orthop Relat Res. 1976;115:4-5.

25. Matsudaira K, Inuzuka K, Kikuchi $\mathrm{N}$, et al. Development of a Japanese version of the Tampa Scale for Kinesiophobia (TSK-J): translation and linguistic validation. Rinsho Seikei Geka. 2013; 48:13-19.

26. Woby SR, Roach NK, Urmston M, Watson PJ. Psychometric properties of the TSK-11: a shortened version of the Tampa Scale for Kinesiophobia. Pain. 2005;117(1):137-144. doi:10.1016/j.pain.2005. 05.029

27. Alghadir AH, Anwer S, Iqbal A, Iqbal ZA. Test-retest reliability, validity, and minimum detectable change of visual analog, numerical rating, and verbal rating scales for measurement of osteoarthritic knee pain. J Pain Res. 2018;11:851-856. doi:10.2147/JPR.S158847

28. Herdman M, Gudex C, Lloyd A, et al. Development and preliminary testing of the new five-level version of EQ-5D (EQ-5D-5L). Qual Life Res. 2011;20(10):1727-1736. doi:10.1007/s11136-011-9903-x

29. Golicki D, Niewada M, Buczek J, et al. Validity of EQ-5D-5L in stroke. Qual Life Res. 2015;24(4):845-850. doi:10.1007/s11136-0140834-1

30. Bilbao A, García-Pérez L, Arenaza JC, et al. Psychometric properties of the EQ-5D-5L in patients with hip or knee osteoarthritis: reliability, validity and responsiveness. Qual Life Res. 2018;27 (11):2897-2908. doi:10.1007/s11136-018-1929-x

31. EQ-5D. Terminology [Homepage on the Internet]. Amsterdam: 2020 EuroQol Research Foundation; 2020. Available from https://euroqol. org/support/terminology/. Accessed August 14, 2020.

32. Shiroiwa T, Ikeda $\mathrm{S}$, Noto $\mathrm{S}$, et al. Comparison of value set based on DCE and/or TTO data: scoring for EQ-5D-5L health states in Japan. Value Health. 2016;19(5):648-654. doi:10.1016/j.jval.2016.03.1834

33. Tavakol M, Dennick R. Making sense of Cronbach's alpha. Int J Med Educ. 2011;2:53-55. doi:10.5116/ijme.4dfb.8dfd

34. Hu L-T, Bentler PM. Cutoff criteria for fit indexes in covariance structure analysis: conventional criteria versus new alternatives. Struct Equ Modeling. 1999;6(1):1-55. doi:10.1080/10705519909540 118

35. Saleh A, Thirukumaran C, Mesfin A, Molinari RW. Complications and readmission after lumbar spine surgery in elderly patients: an analysis of 2320 patients. Spine J. 2017;17(8):1106-1112. doi:10.10 16/j.spinee.2017.03.019

36. Miyagishima K, Tsushima E, Ishida K, Sato S. Factors affecting health-related quality of life one year after lumbar spinal fusion. Phys Ther Res. 2017;20:36-43. doi:10.1298/ptr.E9919

37. Lin EY, Chen PY, Tsai PS, Lo WC, Chiu HY. Trajectory of health-related quality of life and its determinants in patients who underwent lumbar spine surgery: a 1-year longitudinal study. Qual Life Res. 2018;27:2251-2259. doi:10.1007/s11136-018-1888-2

38. Roelofs J, Sluiter JK, Frings-Dresen MHW, et al. Fear of movement and (re)injury in chronic musculoskeletal pain: evidence for an invariant two-factor model of the Tampa Scale for Kinesiophobia across pain diagnoses and Dutch, Swedish, and Canadian samples. Pain. 2007;131:181-190. doi:10.1016/j.pain.2007.01.008

39. Marshall PWM, Schabrun S, Knox MF. Physical activity and the mediating effect of fear, depression, anxiety, and catastrophizing on pain related disability in people with chronic low back pain. PLoS One. 2017;12:e0180788. doi:10.1371/journal.pone.0180788 
40. Brellenthin AG, Crombie KM, Cook DB, Sehgal N, Koltyn KF. Psychosocial influences on exercise-induced hypoalgesia. Pain Med. 2017;18:538-550. doi:10.1093/pm/pnw275

41. Hviid J-CT, Thorlund JB, Vaegter HB. Walking increases pain tolerance in humans: an experimental cross-over study. Scand J Pain. 2019;19(4):813-822. doi:10.1515/sjpain-2019-0070

42. Rice D, Nijs J, Kosek E, et al. Exercise-induced hypoalgesia in pain-free and chronic pain populations: state of the art and future directions. J Pain. 2019;20(11):1249-1266. doi:10.1016/j.jpain.20 19.03 .005

43. Oyibo K, Adaji I, Vassileva J. Social cognitive determinants of exercise behavior in the context of behavior modeling: a mixed method approach. Digit Health. 2018;4:2055207618811555. doi:10. $1177 / 2055207618811555$

44. Lazarus RS, Folkman S. Stress Appraisal and Coping. New York, NY: Springer Publishing Company; 1984.

45. Kasai Y, Suzuki E, Iwase T, Doi H, Takao S, Laks J. Type $\mathrm{D}$ personality is associated with psychological distress and poor self-rated health among the elderly: a population-based study in Japan. PLoS One. 2013;8(10):e77918. doi:10.1371/journal.pone.007 7918

46. Iwata N, Higuchi HR. Responses of Japanese and American university students to the STAI items that assess the presence or absence of anxiety. J Pers Assess. 2000;74(1):48-62. doi:10.1207/S15327752 JPA740104

47. Lindbäck Y, Tropp H, Enthoven P, Abbott A, Öberg B. Prepare: presurgery physiotherapy for patients with degenerative lumbar spine disorder: a randomized controlled trial. Spine J. 2018;18 (8):1347-1355. doi:10.1016/j.spinee.2017.12.009

48. Perkins S, Jenkins LS. Self-efficacy expectation, behavior performance, and mood status in early recovery from percutaneous transluminal coronary angioplasty. Heart Lung. 1998;27(1):37-46. doi:10.1016/s0147-9563(98)90067-x

49. Tsuji H, Tetsunaga T, Tetsunaga T, Nishida K, Misawa H, Ozaki T. The factors driving self-efficacy in intractable chronic pain patients: a retrospective study. J Orthop Surg Res. 2019;14(1):473. doi:10. 1186/s13018-019-1535-9
50. Du S, Hu L, Bai Y, et al. The influence of self-efficacy, fear-avoidance belief, and coping styles on quality of life for Chinese patients with chronic nonspecific low back pain: a multisite cross-sectional study. Pain Pract. 2018;18(6):736-747. doi:10.1111/ papr. 12660

51. Larsson C, Hansson EE, Sundquist K, Jakobsson U. Kinesiophobia and its relation to pain characteristics and cognitive affective variables in older adults with chronic pain. BMC Geriatr. 2016;16 (1):128. doi:10.1186/s12877-016-0302-6

52. Majoor BCJ, Andela CD, Quispel CR, et al. Illness perceptions are associated with quality of life in patients with fibrous dysplasia. Calcif Tissue Int. 2018;102(1):23-31. doi:10.1007/s00223-017-03 29-5

53. Monticone M, Ambrosini E, Vernon H, et al. Efficacy of two brief cognitive-behavioral rehabilitation programs for chronic neck pain: results of a randomized controlled pilot study. Eur J Phys Rehabil Med. 2018;54(6):890-899. doi:10.23736/S1973-9087.18.05206-1

54. Wasser JG, Vasilopoulos T, Zdziarski LA, Vincent HK. Exercise benefits for chronic low back pain in overweight and obese individuals. PM R. 2017;9(2):181-192. doi:10.1016/j.pmrj.2016.06. 019

55. Louw S, Makwela S, Manas L, Meyer L, Terblanche D, Brink Y. Effectiveness of exercise in office workers with neck pain: a systematic review and meta-analysis. S Afr J Physiother. 2017;73 (1):392. doi:10.4102/sajp.v73i1.392

56. Kannan P, Chapple CM, Miller D, Claydon-Mueller L, Baxter GD. Effectiveness of a treadmill-based aerobic exercise intervention on pain, daily functioning, and quality of life in women with primary dysmenorrhea: a randomized controlled trial. Contemp Clin Trials. 2019;81:80-86. doi:10.1016/j.cct.2019.05.004

57. Zronek M, Sanker H, Newcomb J, Donaldson M. The influence of home exercise programs for patients with non-specific or specific neck pain: a systematic review of the literature. J Man Manip Ther. 2016;24(2):62-73. doi:10.1179/2042618613Y.0000000047
Journal of Pain Research

\section{Publish your work in this journal}

The Journal of Pain Research is an international, peer reviewed, open access, online journal that welcomes laboratory and clinical findings in the fields of pain research and the prevention and management of pain. Original research, reviews, symposium reports, hypothesis formation and commentaries are all considered for publication. The manuscript management system is completely online and includes a very quick and fair peer-review system, which is all easy to use. Visit http:// www.dovepress.com/testimonials.php to read real quotes from published authors. 